\title{
BMJ Open Insights into the association of ACEIs/ ARBs use and COVID-19 prognosis: a multistate modelling study of nationwide hospital surveillance data from Belgium
}

José L. Peñalvo (D) , ${ }^{1}$ Els Genbrugge, ${ }^{1}$ Elly Mertens (D) , ${ }^{1}$ Diana Sagastume, ${ }^{1}$ Marianne A B van der Sande, ${ }^{2,3}$ Marc-Alain Widdowson, ${ }^{4}$ Dominique Van Beckhoven (1) , ${ }^{5}$ Belgian Collaborative Group on COVID-19 Hospital Surveillance

To cite: Peñalvo JL, Genbrugge $\mathrm{E}$, Mertens $\mathrm{E}$, et al. Insights into the association of ACEIs/ARBs use and COVID-19 prognosis: a multistate modelling study of nationwide hospital surveillance data from Belgium. BMJ Open 2021;11:e053393. doi:10.1136/ bmjopen-2021-053393

- Prepublication history and additional supplemental material for this paper are available online. To view these files, please visit the journal online (http://dx.doi.org/10.1136/ bmjopen-2021-053393).

Received 13 May 2021 Accepted 25 August 2021
Check for updates

(c) Author(s) (or their employer(s)) 2021. Re-use permitted under CC BY-NC. No commercial re-use. See rights and permissions. Published by BMJ.

For numbered affiliations see end of article.

Correspondence to Professor José L. Peñalvo; jpenalvo@itg.be

\section{ABSTRACT}

Objectives The widespread use of angiotensin-converting enzyme inhibitors (ACEIs) and angiotensin receptor blockers (ARBs) by patients with chronic conditions raised early concerns on the potential exacerbation of COVID-19 severity and fatality. Previous studies addressing this question have used standard methods that may lead to biased estimates when analysing hospital data because of the presence of competing events and event-related dependency. We investigated the association of ACEls/ ARBs' use with COVID-19 disease outcomes using time-toevent data in a multistate setting to account for competing events and minimise bias.

Setting Nationwide surveillance data from 119 Belgian hospitals.

Participants Medical records of 10866 patients hospitalised from 14 March 2020to 14 June 2020 with a confirmed SARS-CoV-19 infection and information about ACEIs/ARBs' use.

Primary outcome measure Multistate, multivariate Cox-Markov models were used to estimate the hazards of patients transitioning through health states from admission to discharge or death, along with transition probabilities calculated by combining the baseline cumulative hazard and regression coefficients.

Results After accounting for potential confounders, there was no discernable association between ACEls/ARBs' use and transfer to intensive care unit (ICU). Contrastingly, for patients without ICU transfer, ACEIs/ARBs' use was associated with a modest increase in recovery (HR 1.07, $95 \% \mathrm{Cl} 1.01$ to $1.13, \mathrm{p}=0.027$ ) and reduction in fatality (HR $0.83,95 \% \mathrm{Cl} 0.75$ to $0.93, \mathrm{p}=0.001$ ) transitions. For patients transferred to ICU admission, no evidence of an association between ACEIs/ARBs' use and recovery (HR $1.16,95 \% \mathrm{Cl} 0.97$ to $1.38, \mathrm{p}=0.098$ ) or in-hospital death (HR $0.91,95 \% \mathrm{Cl} 0.73$ to $1.12, \mathrm{p}=0.381$ ) was observed. Male gender and older age were significantly associated with higher risk of ICU admission or death. Chronic cardiometabolic comorbidities were also associated with less recovery.

\section{STRENGTHS AND LIMITATIONS OF THIS STUDY}

$\Rightarrow$ The study uses nationwide hospital surveillance data, and includes all general hospitals in Belgium.

$\Rightarrow$ The use of a comprehensive database, but more so the utilisation of models adequately fitting timeto-event data with mutually exclusive health states results in less probability of introducing biases and are crucial for correct evidence-based information for decision making.

$\Rightarrow$ Only transfer to intensive care was linked to a calendar date and was therefore the only event which could be used as a proxy for severe disease state in our time-dependent model, indicating that our estimates might represent more of a critical state of the patient.

$\Rightarrow$ Information on the use of angiotensin-converting enzyme inhibitors/angiotensin receptor blockers (ACEls/ARBs) was available at admission only, without any further information on the in-hospital use of ACEIs/ARBs for those patients which could introduce a risk of immeasurable time bias if treatment discontinuation versus continuation has an impact on COVID-19 severity outcomes.

Conclusions For the first time, a multistate model was used to address magnitude and direction of the association of ACEIs/ARBs' use on COVID-19 progression. By minimising bias, this study provided a robust indication of a protective, although modest, association with recovery and survival.

\section{BACKGROUND}

COVID-19 is known to affect more severely the older individuals, men and patients with chronic respiratory or cardiometabolic conditions such as cardiovascular disease (CVD), hypertension (HTN) and diabetes mellitus (DM).$^{1-3}$ Also, common risk factors for chronic conditions, 
such as smoking and particularly obesity, have been identified as key predictors of hospitalisation and critical illness, even in young adults with no underlying conditions. ${ }^{45}$ Although the pathogenesis of certain chronic diseases predisposes to severe COVID-19 outcomes, common chronic medications have also been a concern because of their potential interaction with the angiotensin-converting enzyme 2 (ACE-2) ${ }^{6}$ SARS-CoV-2 binds to target cells using ACE-2 in cell membranes, ${ }^{7}$ an enzyme that physiologically counters the renin-angiotensin-aldosterone system (RAAS) activation, degrading angiotensin II to attenuate its subsequent physiological action. Modulation of the RAAS is a common mode of action of widely used antihypertensive drugs such as angiotensin-converting enzyme inhibitors (ACEIs) and angiotensin II receptor blockers (ARBs) which have been reported to upregulate ACE-2 expression in the heart, and mostly in animal models. ${ }^{8-12}$ This potential upregulation suggested that ACE-2 expression may be increased in patients treated with ACEIs or ARBs, potentially worsening further the prognosis of COVID-19 infection among patients with chronic conditions, and raising early concerns during the first phases of the outbreak of the SARS-CoV-2 pandemic. ${ }^{1314}$ As patients with chronic comorbidities were also identified as more vulnerable to severe COVID-19 disease, it is necessary to understand whether part of this vulnerability could be attributed to the use of ACEIs/ARBs and to evaluate the risk of discontinuing this otherwise essential, first-line therapy, for hypertensive and diabetic patients.

To date, a number of studies addressing the potential effect of ACEIs and ARBs on the prognosis of COVID-19 have been reported, mostly supporting the absence of harmful effects of these drugs on COVID-19 prognosis. ${ }^{15-32}$ In these studies, a wide range of statistical methods have been used to test this hypothesis, including comparison of proportions, percentage points, logistic regression, or time-to-event analysis and Cox models. The use of standard methods for these particular analyses can easily lead to biased estimates, in particular when analysing hospital data because of the presence of competing events, such as death and recovery, and the time-dependency of these competing events. ${ }^{33}$ As such, the analysis of the association of ACEIs/ARBs on the progression of COVID-19 or related mortality requires the assessment of competing risks/events. Analysing time-to-event data in a multistate setting would better fit the true progression of COVID-19 in hospitalised patients, as shown by two studies using a multistate-approach in the context of COVID-19. ${ }^{35}$ Multistate models allow for studying clinically competing events (discharged alive vs deceased), as well as disease progression (eg, in terms of hospital stay duration, transfer to intensive care units (ICUs), treatment received), simultaneously over time. This multistate model framework ensures avoiding bias that stems from censoring patients (informative censoring and/or selection bias) and time-dependent predictors (timedependent bias), as well as circumvent events-related dependency by treating disease progression as a transient state that might influence the probability of experiencing a certain future outcome depending on patient's risk factors. Although accounting for these biases, we revisited the hypothesis of the potential association of ACEIs/ARBs' use in patient's prognosis during hospitalisation using a competing risk multistate model and nationwide hospital surveillance data on patients with COVID-19 in Belgium.

\section{PATIENTS AND METHODS \\ Data sources}

All methods were carried out in accordance with relevant guidelines and regulations. Nationwide hospital surveillance data on patients with COVID-19 in Belgium are routinely gathered by Sciensano, the Belgian Institute of Public Health, which is the institution legally entitled for the surveillance of infectious diseases in Belgium (Royal Decree of 21 March 2018). Retrieving informed consent was determined as a disproportional load on the hospital resources in the crisis situation. An information letter was given to the patients at the time of discharge which contained an explanation of their rights concerning the data that were gathered by Sciensano. The COVID-19 hospital surveillance was authorised by an independent administrative authority protecting privacy and personal data and was approved by the ethical committee of Ghent University Hospital (BC-07507). Details on the Belgian COVID-19 hospital surveillance system have been previously published. ${ }^{37}$ The system covers 119 hospitals in Belgium, who report standardised information on hospitalised patients with COVID-19 collected through a structured questionnaire at hospital admission and discharge. An anonymised subset of data from Sciensano was shared with the Institute of Tropical Medicine through a secured data transfer platform applying data encryption. Ethical clearance was obtained from the Institutional Review Board of the Institute of Tropical Medicine after revision of the research protocol number 1393/20, 02 May 2020.

Variables collected at admission include the date of hospital admission, reason for hospitalisation, symptoms, clinical signs, treatment with ACEIs or ARBs and demographic information such as age, gender and the presence of chronic comorbidities. Information recorded at discharge includes laboratory values, details on COVID-19-specific treatments during hospital stay, date of discharge, health status at discharge and measures on the severity of the disease such as the need for transfer to ICU, invasive ventilation support and/or oxygenation by extracorporeal membrane oxygenation (ECMO), and the development of a bacterial and/or fungal superinfection, pneumonia and/or acute respiratory distress syndrome (ARDS). Dates for these severe events were only available for ICU transfer.

\section{Study population}

Adult patients with a SARS-CoV-19 infection confirmed by PCR, and/or suggestive imaging alterations on chest CT combined with typical clinical presentation, at admission or while hospitalised in Belgium from 14 March 2020 to 14 June 2020 were considered as patients with COVID-19 ( $\mathrm{n}=16$ 341). Of these, patients with completed questionnaires both on admission and discharge (12 109 patients, 74.1\%) were 
selected. Information on patients admitted to hospitals before 1 March $2020(270,1.65 \%)$ for reasons other than COVID-19 and infected while hospitalised was also removed. Furthermore, information on patients with implausible admission dates was removed, including the following: date of discharge before date of admission (42 patients, $0.25 \%$ ); date of ICU transfer preceding date of admission (31 patients, $0.19 \%)$; date of discharge before date of ICU transfer (2 patients, $0.01 \%$ ); date of discharge preceding the date of ICU discharge if the difference was $>1$ day (47 patients, $0.28 \%$ ). The final dataset for descriptive analyses included information on 11717 patients with COVID-19. For the multivariate multistate model, patients with unknown use of ACEIs/ARBs (718 patients, $6.12 \%$ ) were also excluded, along with those missing information on gender (118 patients, $0.72 \%$ ) or unknown transfer to ICU $(15,0.09 \%)$. The final dataset for the multistate model contained information from 103 hospitals in Belgium and 10866 patients with COVID-19, including 539 patients $(5 \%)$ that were admitted directly to ICU.

\section{Study outcomes}

Patients were considered to have recovered when their status at hospital discharge was recorded as 'cured' or 'other'. In the latter case, it was assumed they were allowed to recover at home, revalidation centre or nursing home. Patients were considered as an in-hospital death when their status at discharge was recorded as 'death'. Patients were considered lost to follow-up when their status at discharge was recorded as 'unknown' or when they were transferred to another hospital (recorded as status at discharge $=$ 'transfer to another hospital'), as no further information was available. Severe COVID-19 was captured in the database as an illness that required ECMO or artificial ventilation, or to have experienced ARDS, pneumonia, bacterial and/or fungal co-infection, or required transfer to or treament at ICU. Among these, event date was only available for transfer to ICU, and only this variable could be therefore selected for the models as time-defined severity outcome. Incorporating the intermediate event of ICU transfer allows for a patient's risk profile for recovery and death to be different before and after this intermediate event. In order to do so, time to severe illness was defined as the time passed from the date of hospital admission to the date of transfer to ICU, while length of hospital stay was defined as the date from hospital admission to the date of hospital discharge (either recovery, in-hospital death or lost to follow-up).

\section{Information on ACEls/ARBs and conditions related to COVID-19 prognosis}

A dedicated section in the admission dataset covered the use of ACEIs, ARBs or both, without specification of the specific drug. The admission database contained information on the following factors associated with COVID-19. ${ }^{38}$ Demographics (age and gender), risk factors (current smoking, high blood pressure (HBP) and obesity), prevalent comorbidities (DM, chronic renal disease, CVD, chronic lung disease, chronic liver disease, malignant solid neoplasms, haematological cancers and immunosuppression). Smoking status was only available for $53 \%$ of the patients. Obesity also presented a large number of missing values $(33.2 \%)$ because this variable was recorded only after 3 April 2020. Similarly, there were also missing values for cognitive issues $(5.7 \%)$ as this variable was recorded only after 23 March 2020.

\section{STATISTICAL MODEL}

Patient's characteristics at hospital admission, ICU stay and at hospital discharge were visualised on histograms and summarised as mean scores and SDs or counts and percentages for continuous and categorical variables, respectively. Descriptive analyses were provided for patients overall and stratified by ACEIs/ARBs' use, including unknown use.

To study the association of ACEIs/ARBs on COVID-19 progression on a multivariate multistate model, a first model for identiying confounders was carried out. A backwards stepwise logistic regression with ACEIs/ARBs' use as dependent variable, and including factors and conditions previously associated with COVID-19 outcomes and present in the database, ${ }^{39} 40$ was used to inform the selection of potential confounders based on Akaike's information criterion. The variables used in the variable selection model were gender, age, HBP, CVD, DM, obesity, chronic renal disease, chronic liver disease, chronic lung disease, solid malignant neoplasms, haematological cancers, immunodepression and cognitive impairment. Two models were used depending on the availability of data, a first model (model 1) excluding variables collected at a later date (obesity and cognitive issues) and using a full dataset $(\mathrm{n}=10$ 866), and a complete case analysis (model 2) excluding patients with missing data for obesity and cognitive issues $(\mathrm{n}=7303)$.

We devised a multistate model reflecting the progression of patients from hospital admission to discharge accounting for the patient's characteristics identified to be potential confounders, and introducing ACEIs/ARBs use as dependent variable. The model starts with one initial state (hospitalisation), a potential (transient) state defined as ICU transfer (as a proxy for severe COVID-19 disease, as only ICU transfer had an associated date in the database) and two absorbing states (in-hospital death and recovery). The multistate model is characterised by transition hazards between the states; defined as the instantaneous risk for moving from one health state to another. The transitions hazards are used to calculate transition probabilities, as the conditional probabilities of experiencing future outcomes, given the history and a particular set of prognostic factors (model covariates) for a given patient. The four-state model thereby comprised the following five possible patient's transitions: (1) hospitalisation to ICU, (2) hospitalisation to recovery, (3) hospitalisation to in-hospital death, (4) ICU to recovery and (5) ICU to in-hospital death, as presented in figure 1. A Cox-Markov model for the regression on the transition-specific hazards was fitted using the coxph and msfit functions in R survival package. ${ }^{41}$ This approach is equivalent of constructing five separate Cox regression models, one for each transition hazard. The cumulative baseline transition hazard (all covariate values 


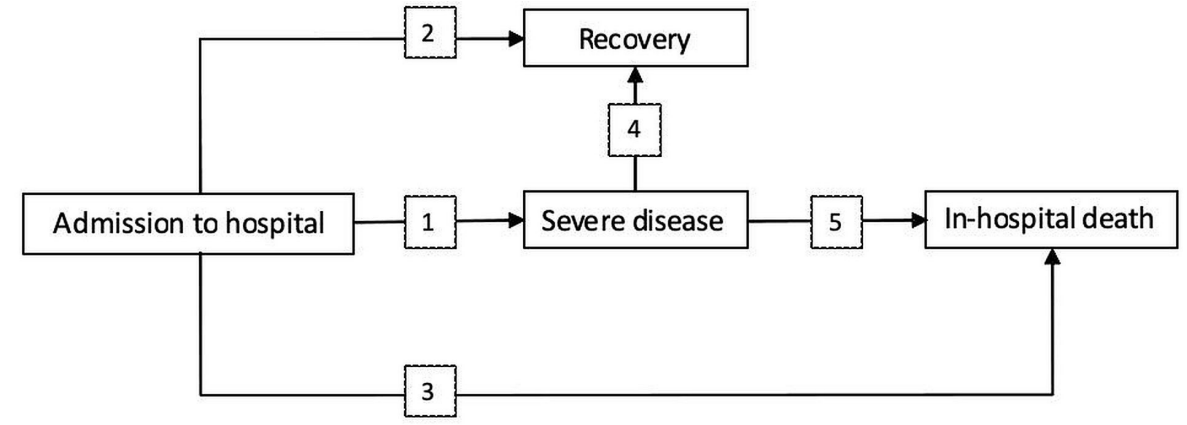

\begin{tabular}{|c|c|c|c|c|c|c|}
\hline From & $\begin{array}{l}\text { To } \\
\text { Admission }\end{array}$ & Severity & Recovery & Death & No event & Total \\
\hline Admission & 0 & $863(8.4)^{1}$ & $7633(73.9)^{2}$ & $1738(16.8)^{3}$ & $93(0.9)$ & 10327 \\
\hline Severity & 0 & 0 & $817(58.3)^{4}$ & $468(33.4)^{5}$ & $117(8.3)$ & $1402^{*}$ \\
\hline Recovery & 0 & 0 & 0 & 0 & $8450(100)$ & 8450 \\
\hline Death & 0 & 0 & 0 & 0 & 2206 (100) & 2206 \\
\hline
\end{tabular}

Figure 1 Schematic representation of the competing risk multistate model and transition event matrix (number (\%) patients in each transition. Numbers in superscript represent transitions depicted in the figure. ${ }^{\star} 539$ patients were directly admitted to ICU. ICU, intensive care unit.

equal to the reference value) was estimated by the Breslow estimator with the Aalen estimator of variance. ${ }^{42}$ We integrated these separate Cox models in a multistate framework studying different outcomes simultaneously and allowing the calculation of transition probabilities. The transition probabilities were then estimated by combining the baseline cumulative hazard and regression coefficients. Using $\mathrm{R}$, the mstate package and msfit function were applied to obtain cumulative (baseline) transition hazards and the function probtrans to obtain the transition probabilities. ${ }^{43}$ Estimates obtained from the Cox-Markov models are displayed in a table and significance is established at the $5 \%$ significance level. Cumulative (baseline) transition hazard plots and transition probability plots were also generated for visual aid. In a setting with covariates, a regression model for the transition specific hazards was used, whereby the covariates may help to explain the difference in transition hazards. Model diagnostics were performed to check model assumptions of proportional hazards, linearity and interactions. Assumptions to the Markov model were assessed by including time from hospital admission to ICU transfer in the model for transitions 4 and 5. A relaxation of the Markov assumption was also explored in the analysis.

\section{Patient and public involvement}

As a secondary data analysis of COVID-19 surveillance data, this study did not involve patients or the public in the design, conduct or dissemination plans.

\section{RESULTS}

\section{Descriptive analysis}

From the 11717 patients available for this analysis, almost of all them $(94.2 \%)$ presented symptoms or clinical signs compatible with COVID-19 at admission (online supplemental table 1). Most frequent symptoms were fever $(61.3 \%)$ and cough $(53.2 \%)$, and most frequent signs were abnormal pulmonary imaging $(63.1 \%)$ compatible with viral pneumonia, abnormal auscultation (44.8\%) and dyspnoea $(42.4 \%)$. On admission, $15.1 \%$ of patients had a record of taking ACEIs and $8.5 \%$ ARBs, with only $0.4 \%$ taking both ACEIs and ARBs. For the purpose of this analysis, these patients were merged as users of ACEIs/ ARBs. The majority of patients (69.9\%) were nonusers of ACEIs/ARBs versus $23.9 \%$ of users, and only for a small proportion $(6.1 \%)$ of patients the use of ACEIs/ARBs was unknown (table 1). No difference was seen in the frequency of signs and symptoms reported according to ACEIs/ARBs' use (online supplemental table 1). Patients using ACEIs/ARBs were markedly older (median (IQR) age 76 (65-84) years) than non-users (67 (53-81) years) while no gender differences were observed. As expected, users of ACEIs/ ARBs presented more frequently (74.4\%) HBP than non-users of ACEIs/ARBs (39.2\%), as well as chronic lung disease $(16.8 \%$ vs $14.4 \%)$, chronic renal disease (19.3\% vs $11.1 \%)$, DM $(33.3 \%$ vs $18.1 \%)$ and particularly CVD (53.1\% vs $28.4 \%)$. Multiple comorbidities (HBP, DM and CVD) were more frequent among users of ACEIs/ARBs (15.1\%) than non-users $(4.9 \%$; table 1). During hospital stay, $>80 \%$ of patients with COVID-19 experienced one severe episode of either pneumonia, superinfection, ARDS or mechanical ventilation, and $25.9 \%$ of patients had two or more severe episodes (table 2). The most common manifestation of COVID-19 severity was pneumonia $(79.1 \%)$, followed by other infections (19.4\%), ARDS (12.7\%) and artificial ventilation $(7.6 \%)$. Frequency of severe conditions was nearly the same for both users of ACEIs/ ARBs and non-users (27.8\% vs $25.4 \%)$. Of all admitted patients, $1518(13.0 \%)$ were transferred to ICU, mostly those with severe pneumonia $(93.7 \%)$, or in need of 
Table 1 Characteristics of patients with COVID-19 at hospital admission according to ACEIs/ARBs' use

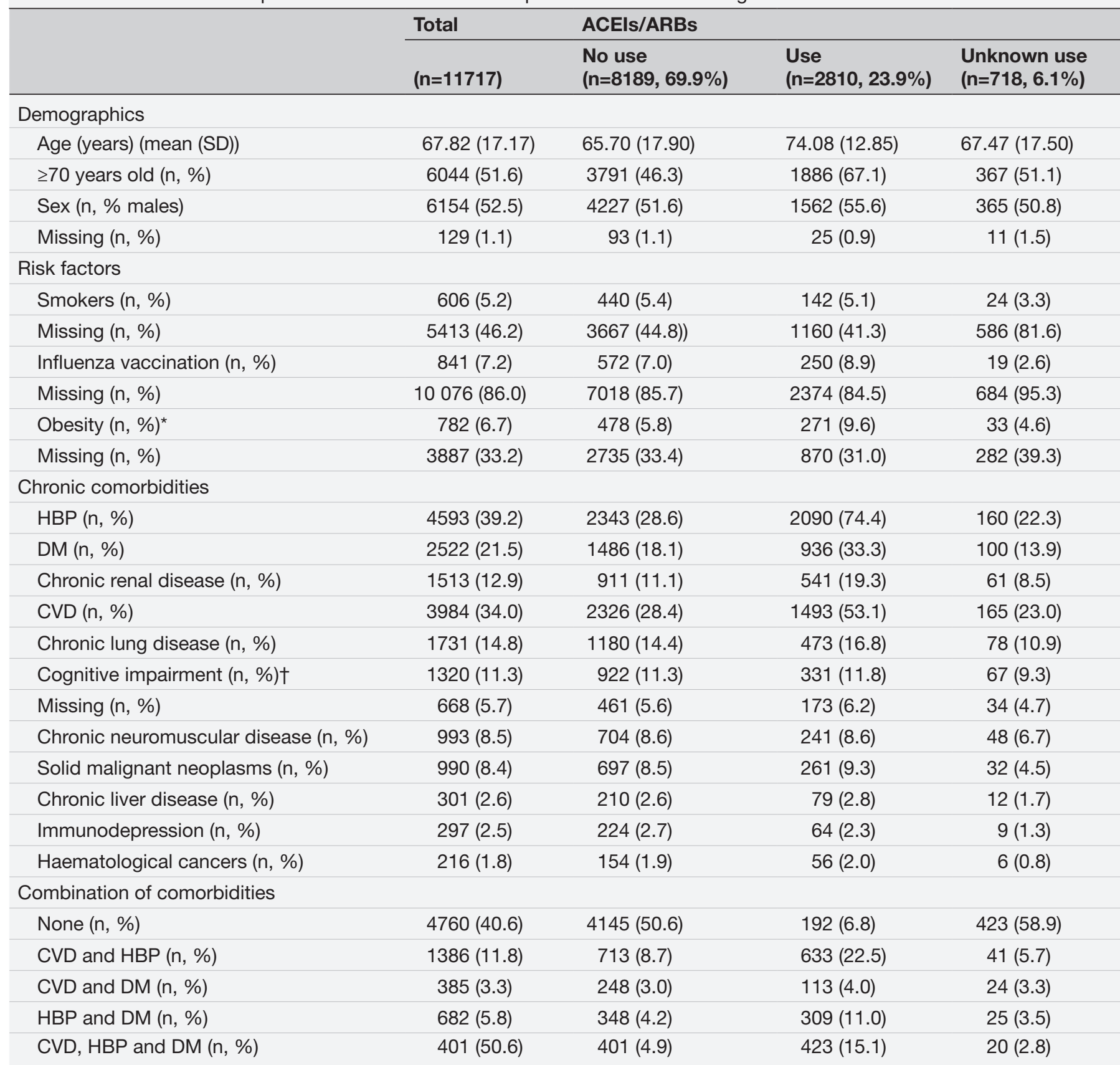

*Values collected only after 3 April 2020.

†Values reported only after 23 March 2020.

ACEIs/ARBs, ACE inhibitors/angiotensin receptor blockers; CVD, cardiovascular disease; DM, diabetes mellitus; HBP, high blood pressure.

artificial ventilation $(58.0 \%)$, and remained at ICU for a mean duration of $11.5 \pm 10.7$ days. Transfer to ICU was marginally more frequent among users of ACEIs/ ARBs $(15.1 \%$ vs $12.1 \%)$. Almost $78 \%$ of the patients admitted to Belgian hospitals recovered from COVID19 , either during hospitalisation $(51.2 \%)$ or at home or revalidation centre or nursing home $(26.4 \%)$ after an average of $12.6 \pm 10.9$ days in the hospital. Only $2 \%$ of patient's information was lost to follow-up (transferred to another healthcare provider or unknown status at discharge).

\section{Multistate model}

A multivariate state-arrival-extended Cox-Markov model was used to study the potentially different progression of patients with COVID-19 through health states during hospitalisation according to the use of ACEIs/ARBs. Possible transitions and number of patients in each health state are represented in figure 1 . The selection of variables for adjusting the models was based on backwards stepwise logistic regression of ACEIs/ARBs' use as a function of potential confounding factors associated with COVID-19 recorded at admission (online supplemental table 2). A 
Table 2 Frequency of severity events among patients with COVID-19 during hospital stay and recorded outcomes at discharge according to ACEIs/ARBs' use at admission

\begin{tabular}{|c|c|c|c|c|}
\hline & \multicolumn{3}{|c|}{ ACEls/ARBs } & \multirow[b]{2}{*}{ Unknown use } \\
\hline & Total & No use & Use & \\
\hline & $(n=11717)$ & $(n=8189,69.9 \%)$ & $(n=2810,23.9 \%)$ & $(n=718,6.1 \%)$ \\
\hline \multicolumn{5}{|l|}{ Severe conditions } \\
\hline Pneumonia (n, \%) & $9265(79.1)$ & $6501(79.4)$ & $2260(80.4)$ & $504(70.2)$ \\
\hline Missing (n, \%) & $532(4.5)$ & $372(4.5)$ & $73(73)$ & $87(87)$ \\
\hline Superinfection (n, \%) & 2268 (19.4) & $1548(18.9)$ & $589(21.0)$ & $131(18.2)$ \\
\hline Missing (n, \%) & $1277(10.9)$ & $820(10.0)$ & $320(11.4)$ & $137(19.1)$ \\
\hline $\operatorname{ARDS}(\mathrm{n}, \%)$ & $1492(12.7)$ & $996(12.2)$ & $389(13.8)$ & $107(14.9)$ \\
\hline Missing (n, \%) & $1047(8.9)$ & $671(8.2)$ & $263(9.4)$ & $113(15.7)$ \\
\hline Mechanical ventilation (n, \%) & $893(7.6)$ & $571(7.0)$ & $249(8.9)$ & 73 (10.2) \\
\hline Missing (n, \%) & $627(5.4)$ & $383(4.7)$ & $170(6.0)$ & $74(10.3)$ \\
\hline \multicolumn{5}{|l|}{ Number of severe conditions (n, \%) } \\
\hline None & $2143(18.3)$ & $1492(18.2)$ & $465(16.5)$ & $186(25.9)$ \\
\hline One & $6537(55.8)$ & $4620(56.4)$ & $1565(55.7)$ & $352(49.0)$ \\
\hline Two or more & $3037(25.9)$ & $2077(25.4)$ & $780(27.8)$ & $180(25.1)$ \\
\hline \multicolumn{5}{|l|}{ Intensive care } \\
\hline Transfer to ICU (n, \%) & $1518(13.0)$ & $990(12.1)$ & $425(15.1)$ & $103(14.3)$ \\
\hline Missing (n, \%) & $16(0.1)$ & $16(0.2)$ & $0(0)$ & $0(0)$ \\
\hline Transfer to ICU +pneumonia (n, \%) & $1423(93.7)$ & $932(94.1)$ & $395(92.9)$ & $96(93.2)$ \\
\hline Transfer to ICU +superinfection (n, \%) & $653(43.0)$ & $423(42.7)$ & $181(42.6)$ & $49(47.6)$ \\
\hline Transfer to ICU +ARDs (n, \%) & $831(54.7)$ & $547(55.3)$ & $224(52.7)$ & $60(58.3)$ \\
\hline Transfer to ICU +mechanical ventilation (n, \%) & $880(58.0)$ & $561(56.7)$ & $246(57.9)$ & $73(70.9)$ \\
\hline Length (days) of ICU stay (mean (SD)) & $11.5(10.7)$ & $11.415(10.7)$ & $11.3(10.8)$ & $12.9(11.0)$ \\
\hline \multicolumn{5}{|l|}{ Discharge status } \\
\hline Recovered at discharge (n, \%) & $6003(51.2)$ & $4244(51.8)$ & $1378(49.0)$ & $381(53.1)$ \\
\hline Recovered at home (n, \%) & $3093(26.4)$ & 2201 (26.9) & $722(25.7)$ & $170(23.7)$ \\
\hline In-hospital death (n, \%) & $2388(20.4)$ & $1574(19.2)$ & $622(23.6)$ & $152(21.2)$ \\
\hline Transferred (n, \%) & 201 (1.7) & $149(51.8)$ & $44(49.0)$ & $8(53.1)$ \\
\hline Unknown (n, \%) & $32(0.3)$ & $21(0.3)$ & $4(0.1)$ & $7(1.0)$ \\
\hline Length (days) of hospital stay (mean (SD)) & $12.6(10.9)$ & $12.1(10.5)$ & $13.9(11.7)$ & $12.2(11.6$ \\
\hline
\end{tabular}

ACEIs/ARBs, ACE inhibitors/angiotensin receptor blockers; ARDS, acute respiratory distress syndrome; ICU, intensive care unit.

first model (model 1) using all available patients identified the following five variables associated with the use of ACEIs/ARBs and COVID-19: male gender (OR 1.33, $95 \%$ CI 1.21 to 1.47 ), older age (OR $1.01,95 \%$ CI 1.01 to 1.02 per 1 year increase), prevalent CVD (OR 1.71, 95\% CI 1.55 to 1.90 ), diabetes (OR $1.38,95 \%$ CI 1.24 to 1.54 ) and HBP (OR $5.65,95 \%$ CI 5.10 to 6.27 ). Additionally, a second model (model 2) was used in sensitivity analysis including additional covariates (prevalent obesity and cognitive impairment) that were only available in a subset (62\%) of patients (online supplemental table 2). Because very few patients were asymptomatic on admission it was deemed unnecessary to adjust the regression models for severity of disease at admission. For 16 patients $(0.1 \%)$, it was unknown whether they were transferred to ICU.
These patients are therefore excluded from the multistate Cox-Markov regression analysis.

Plots for the cumulative hazard and transition probability between health states considering ACEIs/ARBs' use were obtained by setting all model covariates to reference values (female gender, no CVD, no HBP and no DM), and median age (70 years; figure 2 ). When looking at the cumulative hazard for the five possible transitions (figure 2A), the hazard for recovery was markedly greater than that of in-hospital death. In comparison with the other cumulative hazards, the hazard for transfer to ICU was substantially smaller, representative of most patients with COVID-19 not needing intensive care, or not meeting criteria for admission (for instance, after evaluation of fraility,and chance of survival). Transfer to ICU was 

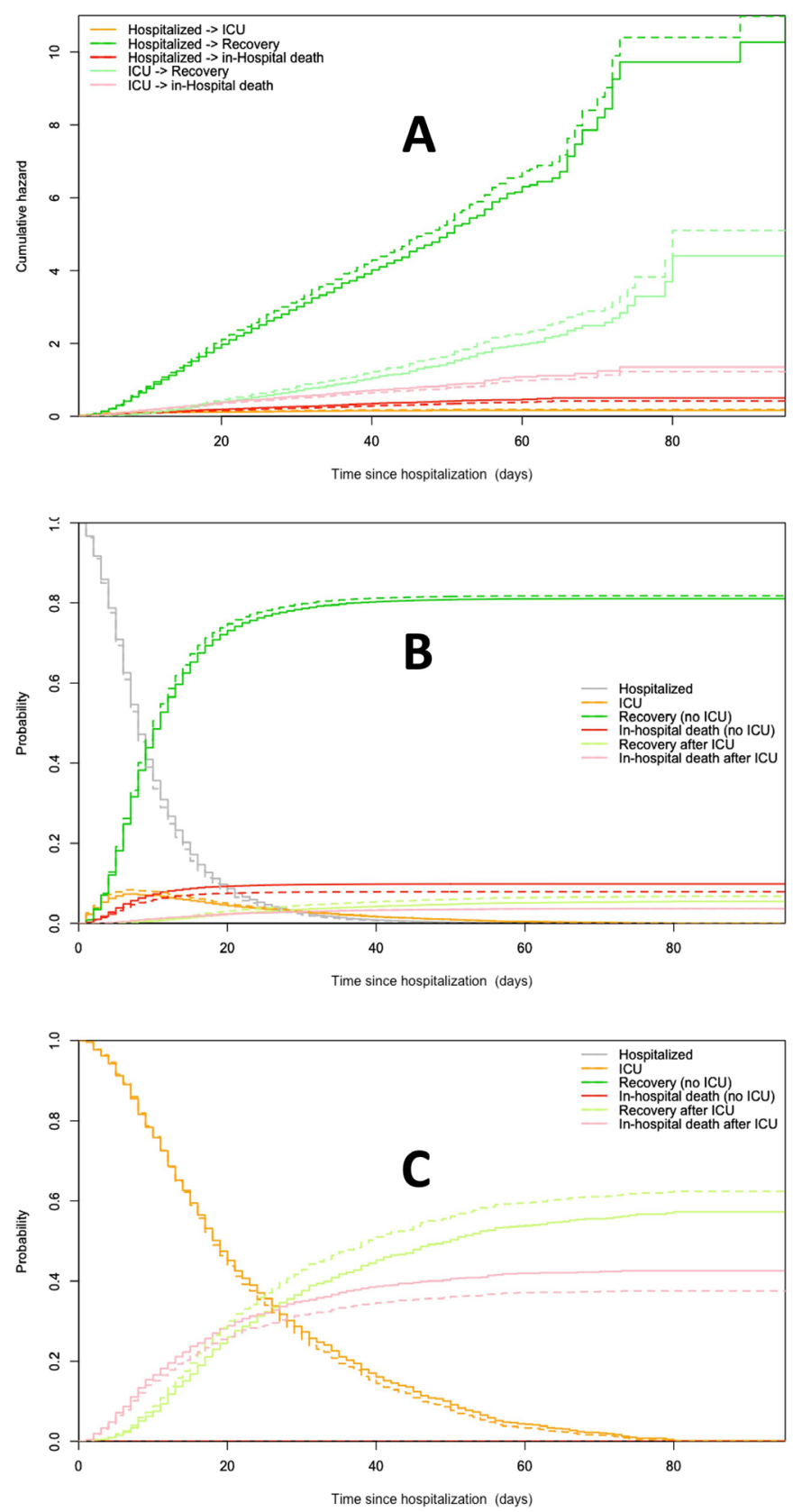

Figure 2 Plots for (A) cumulative transition hazards, (B) state transition probabilities and $(C)$ transition probabilities after transfer to intensive care in a multistate competing risk model considering ACEls/ARBs' use (dashed line) versus no use (solid line). ACEls/ARBs, ACE inhibitors/angiotensin receptor blockers; ICU, intensive care unit.

associated with an increased hazard for in-hospital death and reduced hazard for recovery. The use of ACEIs/ ARBs was associated with a modest but significant association with the hazard of transitions 2 (more recovery) and 3 (less in-hospital death), from admission. The use of ACEIs/ARBs was not observed to be associated with transfer to ICU (transition 1) or with recovery (transition 4) or in-hospital death (transition 5) after ICU. Overall, the probability of being transferred to ICU was, for most patients, less than that of recovery (figure 2B). However, those needing ICU had a reduced probability of recovery and greater probability to decease in the hospital than those patients not transferred to ICU (figure 2C).

The estimates for the transition hazards for ACEIs/ ARBs' use accounting for identified confounding in the potential association with COVID-19 severity/fatality are presented in table 3. In multivariate models, the use of ACEIs/ARBs was associated (HR 1.07, 95\% CI 1.01 to 1.13 ) with more recovery and less death $(0.83,0.75$ to 0.93$)$. Even though there was a significant association between ACEIs/ARBs' use, the hazard of more recovery (transition 2) and less in-hospital death (transition 3), this effect is modest, especially when reviewing state-occupation probabilities (online supplemental figure 1). In respect to the adjusting variables (online supplemental table 3), male gender and HBP were associated with transfer to ICU (severity), and older age also influenced this transition. Similarly, male gender and older age, as well as prevalent CVD, HBP or DM were associated with less recovery. Similar to transfer to ICU, progression to death was associated with male gender and age, as well as with prevalent CVD. No other comorbidity included in this model (ie, associated with ACEIs/ARBs' use) appeared to be associated with fatality. For severely ill patients (transferred to ICU), recovery or death depended mostly on age, although fatal COVID-19 was also associated with the presence of DM, and a lengthier period between admission and ICU was significantly associated with less recovery after ICU (online supplemental table 3). The impact of further adjustment for variables identified during confounder selection (obesity and cognitive issues) in the state transition of patients with COVID-19 during hospitalisation, resulted in loss of more than half of all patients due to missing values (table 1). Although estimated hazards for previous factors remained similar, the presence of cognitive issues was statistically and significantly associated with transitions 1-3 (ie, less transfer to ICU, less recovery and more in-hospital death), and obesity was strongly and statistically significantly associated with transition 1 only (more transfer to ICU). In this complete case model, after additional adjustment for obesity and cognitive issues, the HR for ACEIs/ARBs use for transition 2 (admission to recovery) did not remain significant probably due to a decreased statistical power, since the point estimates were similar.

\section{DISCUSSION}

In this study, a competing risk multistate model has been developed for the first time to address the magnitude and direction of the association of ACEIs/ARBs' use in COVID-19 prognosis. Our analyses indicate a protective association of ACEIs/ARBs' use, with increased recovery and survival, once important confounding factors such as age, particularly $\geq 70$ years and male gender are accounted for. Chronic comorbidities such as CVD, HBP and DM are also associated with less recovery in this model setting. Although there is a protective association of ACEIs/ARBs' use on COVID-19 in-hospital death 
Table 3 State-arrival-extended Cox-Markov multivariate model's transition HRs (95\% Cl) as a function of ACEls/ARBs

\begin{tabular}{llllll}
\hline \multirow{2}{*}{ Transition } & & ACEls/ARBs' use & & \\
\cline { 3 - 5 } & & Model 1 & P value & Model 2 & P value \\
\hline 1 & Admission $\rightarrow$ Severity & $1.15(0.98$ to 1.36$)$ & 0.092 & $1.10(0.88$ to 1.36$)$ & 0.409 \\
\hline 2 & Admission $\rightarrow$ Recovery & $1.07(1.01$ to 1.13$)$ & 0.027 & $1.05(0.98$ to 1.13$)$ & 0.182 \\
\hline 3 & Admission $\rightarrow$ Death & $0.83(0.75$ to 0.93$)$ & 0.001 & $0.80(0.70$ to 0.91$)$ & 0.001 \\
\hline 4 & Severity $\rightarrow$ Recovery & $1.16(0.97$ to 1.38$)$ & 0.098 & $1.16(0.93$ to 1.45$)$ & 0.195 \\
\hline 5 & Severity $\rightarrow$ Death & $0.91(0.73$ to 1.13$)$ & 0.381 & $1.11(0.83$ to 1.49$)$ & 0.485
\end{tabular}

Model 1: adjusted for gender, age (years), prevalent CVD, HBP, DM and time (days) to severity and model 2: further by prevalent obesity and cognitive issues.

ACEIs/ARBs, ACE inhibitors /angiotensin receptor blockers; CVD, cardiovascular disease; DM, diabetes mellitus; HBP, high blood pressure.

and more recovery, this association is modest, especially when looking at the state-occupation probabilities. In our model, once the patient progresses to a severe state, no association of ACEIs/ARBs' use was observed in the transition probabilities to recovery or in-hospital death; only older age and prevalent DM, remained significant covariates in our model, arguably because of the smaller sample size (transfer to ICU occurred only for $13 \%$ of patients). Previous studies using the same data source identified other comorbidities as independent risk factors for COVID-19 severity/death in ICU patients, including chronic pulmonary disease, chronic renald disease and immunosuppression. ${ }^{44}$ Although we accounted for these factors in our model selection, they were not selected as they are not considered to be related to the use of ACEIs/ ARBs but may nonetheless constitute an independent risk factors for severity.

Because of the important clinical relevance, there have been numerous reports on studies of the potential association between ACEIs/ARBs and (worse) prognosis of COVID-19. Early studies of smaller sample size and mostly descriptive design pointed to either no association or moderately lower rates of severe disease among users of ACEIs/ARBs. ${ }^{15-20}$ Further retrospective analysis involving larger patient samples generally reported a lack of association. ${ }^{21}$ A population-based study in Italy's Lombardy region involving 6272 cases identified across the Regional Health Service and matched 1:5 to population-based control found no association between the use of ACEIs (adjusted OR 0.91, 95\% CI 0.69 to 1.21) or ARBs (adjusted OR 0.83, 95\% CI 0.63 to 1.10 ) and severe/fatal COVID-19. ${ }^{22}$ Similarly, a case-control study in the Spanish region of Madrid with data on 1139 hospitalised cases matched 1:10 to population controls found no association (adjusted OR $0.94,95 \%$ CI 0.77 to 1.15 ) of ACEIs/ARBs' use and severe or fatal disease. ${ }^{23}$ Analysing data from patients in the New York University Langone Health electronic health record who had COVID-19 test results (12 594 patients), neither an increased likelihood of a positive test nor a severe disease status was observed for patients using ACEIs/ARBs (or any other RAAS medication) using propensity score matching. ${ }^{24}$ In a nationwide study in Korea using insurance claims of 66
793 individuals tested for COVID-19, the use of ACEIs/ ARBs was not associated with a higher risk of mortality (adjusted OR $0.88,95 \%$ CI 0.53 to 1.44$).{ }^{25}$ Similarly, a large retrospective analysis of an Italian registry cohort including 43000 patients concluded that the use of neither ACEIs (adjusted HR 0.97, 95\% CI 0.89 to 1.06 ) nor ARBs (adjusted HR 0.98, 95\% CI 0.89 to 1.06 ) was associated with either an increased or decreased risk of all-cause mortality. ${ }^{26}$ A multicenter study with 1128 hypertensive patients, and using mixed-effect Cox models (site as a random effect, and model adjusted for age, gender, comorbidities and in-hospital medications), reported a lower risk for all-cause mortality in the patients using ACEIs/ARBs versus the non-ACEIs/ ARBs group (adjusted HR 0.42, 95\% CI 0.19 to 0.92), and further compared with the use of other antihypertensive drugs (adjusted HR $0.30,95 \%$ CI 0.12 to 0.70 ). ${ }^{27}$ Previous studies using Cox models also reported a reduced mortality risk for patients using ACEIs/ARBs. ${ }^{27} 28$ In others, although not statistically significant, estimates were very similar to the ones reported in our study for mortality (adjusted HR $0.83,95 \%$ CI 0.67 to 1.03 ) and for severe disease (adjusted HR 1.15, 95\% CI 0.95 to 1.41). ${ }^{29}$ Similarly, but outside of the hospital setting, studies with data from general practitioners in England, found a strong association of ACEIs/ARBs' use and a reduced risk of COVID-19 disease (HR 0.63, 95\% CI 0.59 to 0.67 ) although not severity (HR $1.02,95 \%$ CI 0.83 to $1.25)$, and marked interactions with ethnicity with higher risks observed for black African patients compared with white patients. ${ }^{30}$ Variations observed between different ethnicities raise the possibility of specific associations of ACEIs/ARBs on COVID-19 disease susceptibility and severity which deserves further study. Furthermore, three review papers on the topic have concluded there is either no difference or a reduced risk when looking at mortality and/or severe disease, ${ }^{213132}$ and no evidence to support discontinuing the treatment with ACEIs/ARBs. ${ }^{45}$ This substantial body of evidence seems aligned with recent findings from clinical studies that do not support the hyphothesis of an increased expression of ACE-2 in patients with chronic conditions treated with ACEIs or ARBs as a driver of severe COVID-19. ${ }^{10-12}$ 
Whereas the results on an increased risk of severe/ fatal COVID-19 in association with the outpatient use of ACEIs/ARBs appear to point in the same direction, studies on the potential role of in-hospital use of ACEIs/ARBs have described a protective association of continuing the treatment throughout hospitalisation. ${ }^{27} 4647$ In a multicentre study including 1128 adult patients with HTN and diagnosed with COVID-19, including 188 using ACEIs/ARBs and 940 without using ACEIs/ARBs during hospitalisation, the risk for all-cause mortality was lower in the ACEIs/ARBs group versus the non-ACEIs/ARBs group (HR 0.42, $95 \%$ CI 0.19 to 0.92 ). ${ }^{27}$ Similarly, a study conducted in 347 patients hospitalised for COVID-19 in Paris (France) analysing the association between in-hospital exposure of ACEIs/ARBs and mortality within 30 days of hospital admission using logistic regression analysis, no association (OR 0.62, 95\% CI 0.25 to 1.48 ) based on chronic exposure but a protective association (OR $0.25,95 \%$ CI 0.09 to 0.65 ) based on in-hospital exposure was observed. ${ }^{46}$ Among 397 patients with COVID-19 admitted to hospitals in Rozzano-Milan (Italy), the risk of mortality was significantly reduced in patients who continued ACEIs/ARBs as compared with those who discontinued and those not taking ACEIs/ ARBs therapy (OR 0.14, 95\% CI 0.03 to 0.66$).{ }^{47}$ Using data from seven hospitals in Madrid (Spain), no difference in mortality rates was observed among patients that discontinued (340 patients) ACEIs/ARBs treatment (HR $1.01,95 \%$ CI 0.70 to 1.46 ) versus those that continued (280 patients). ${ }^{48}$ Furthermore, recent data from two randomised trials could not confirm any impact on clinical outcomes in hospitalised patients with COVID-19 discontinuing treatment of ACEIs or ARBs as compared with those continuing their treatment, ${ }^{49}{ }^{50}$ hereby further supporting a safe ACEIs/ARBs treatment continuation if there is an indication for treatment.

Our study builds on these previous reports where standard statistical models were used for analysis, introducing a model framework overcoming the risk of biases. ${ }^{33}$ Logistic-based regression models might introduce selection bias by excluding patients who are still hospitalised at the last follow-up, hence the need for time-to-event models that allow patient censoring. ${ }^{33}$ These time-toevent models, such as Cox regression models, should preferably account for the presence of competing risks to avoid informative censoring bias, and for time-dependent predictors to appropriately handle index time or follow-up time of covariates. ${ }^{33}$ Integrating standard Cox models into a multistate framework allows the study of separate outcomes simultaneously and allows the calculation of the transition probabilities, adding a layer of interpretation. In this way, by incorporating event-related dependency, that is, transitions to intermediate events that likely influence disease progression, the multistate model more accurately describes the evolution of COVID-19 in hospitalised patients. ${ }^{51}$ In this study, we used a time-to-event analysis in a multistate framework considering competing risks to account appropriately for censoring, ${ }^{52}$ thereby robustly showing a modest, yet significant, positive association of ACEIs/ARBs' use in recovery and survival of hospitalised patients with COVID-19 accounting for confounding factors.

Our study uses nationwide hospital surveillance data, with mandatory participation, and includes all general hospitals (including university hospitals) in Belgium, both those managed by a public authority and private are represented. The surveillance does not cover psychiatric hospitals or specialist hospitals. ${ }^{37}$ The use of comprehensive datasets, but more so the utilisation of models adequately fitting to time-to-event hospital data with mutually exclusive health states results in less probability of introducing biases and are crucial for correct evidence-based information for decision making. Our study makes some assumptions, and unknowns such as the lack of information on ACEIs/ARBs exact indication and whether their use was continued after admission. This lack of accounting for time-varying exposure introduces a risk of immeasurable time bias, ${ }^{34}$ as seen in many reports, though assumed to be minimal because no difference in COVID-19 severity outcomes between treatment discontinuation versus continuation. ${ }^{49}{ }^{50}$ Our models are not adjusted for severity at baseline since we reasoned that hospital admission was already an indicator of severe disease and $94 \%$ of patients had symptoms compatible with COVID-19 diagnosis at baseline. Furthermore, even though other events potentially indicating severity (ECMO, ARDS, pneumonia, bacterial and/or fungal co-infection) were available in the database, only transfer to ICU was linked to a calendar date and was therefore the only event which could be used as a proxy for severe health state in our time-dependent model, indicating that our estimates might represent more a critical state of the patient. In addition, admission to intensive care is not solely based on the clinical status of the patient, but also on other criteria such as frailty. Also, ICU admission criteria might have been more restrictive in the peak period of the epidemic while certain ICUs were overloaded. Because the surveillance data are limited to the most important variables, we cannot discard the possibility of some degree of residual confounding in our results. An important limitation of our main analysis is the impossibility of adjusting our models for smoking status, obesity and cognitive issues at baseline. Using available smoking information was not deemed appropriate due to the excessive number of missing values, and the lack of information of the reason for the incomplete data. We used, however, data on obsesity and cognitive issues, whose collection was introduced later, in a complete case analysis to confirm the results obtained in the main model. Nevertheless, these analyses on a reduced sample of patients should be interpreted with caution as a time effect is likely present because of the late data collection. Finally, our analyses are based on patient's medical files and rely on how clinicians reported clinical observations and anamnesis which might vary across hospitals, and are representative of the first so-called wave of the epidemic 
in Belgium, and associations might differ in subsequent studies and in other settings.

\section{CONCLUSIONS}

After adjustment for important confounders there is modest, yet significant, positive association of ACEIs' / ARBs' use on recovery and survival of hospitalised patients with COVID-19, without affecting admission to intensive care. This supports the use of ACEIs/ARBs in those patients who need them, also when needing hospitalisation from COVID-19. These findings are based on an analytical model that adequately fits hospital data, where patients progress across different, competing, health states providing a more complete and acurate view of the research question within a reduced risk of bias framework. Integrating standard cox models into a multistate framework allows the study of separate outcomes simultaneously and allows the calculation of the transition probabilities, adding a layer of interpretation. Multistate models should be favoured over separate survival analysis when competing risks are present, and traditional methods such as logit functions should be discouraged when time-to-event is available.

\section{Author affiliations \\ ${ }^{1}$ Unit of Non-Communicable Diseases, Department of Public Health, Institute of Tropical Medicine, Antwerpen, Belgium \\ ${ }^{2}$ Department of Public Health, Institute of Tropical Medicine, Antwerp, Belgium ${ }^{3}$ Global Health Julius Centre for Health Sciences and Primary Care, University Medical Centre Utrecht, Utrecht, Netherlands \\ ${ }^{4}$ Institute of Tropical Medicine, Antwerp, Belgium \\ ${ }^{5}$ Department of Epidemiology and Public Health, Sciensano, Brussels, Belgium}

Correction notice This article has been corrected since it first published. The collaborator group 'Belgian Collaborative Group on COVID-19 Hospital Surveillance' has been added in the author byline.

\section{Twitter José L. Peñalvo @JosePenalvo}

Collaborators The Belgian Collaborative Group on COVID-19 Hospital Surveillance consists of all local investigators who are responsible for the design of the COVID surveillance, identifying variables for inclusion, and data collection in each of the hospitals. They have all approved the final manuscript.Samy Amir Aouachria, Centre Hospitalier Chrétien, Liège, Belgium; Kristof Bafort, Mariaziekenhuis, Pelt, Belgium; Leilla Belkhir, Cliniques Universitaires Saint-Luc, Brussels, Belgium; Koen Blot, Sciensano, Belgium; Nathalie Bossuyt, Sciensano, Belgium; Vincent Colombie, Centre Hospitalier Epicura, Baudour, Belgium; Nicolas Dauby, Centre Hospitalier Universitaire Saint-Pierre, Brussels, Belgium; Paul De Munter, Universitair Ziekenhuis Leuven, Leuven, Belgium; Robby De Pauw, Sciensano, Brussels, Belgium; Didier Delmarcelle, Clinique St. Jean, Brussels, Belgium; Mélanie Delvallee, Centre Hospitalier de Wallonie Picarde, Tournai, Belgium; Rémy Demeester, Centre Hospitalier Universitaire de Charleroi, Charleroi, Belgium; Thierry Dugernier, Clinique Saint-Pierre, Ottignies, Belgium; Xavier Holemans, Grand Hôpital de Charleroi, Charleroi, Belgium; Benjamin Kerzmann, Clinique Notre Dame de Grâce, Gosselies, Belgium; Pierre Yves Machurot, Centre Hospitalier de l'Ardenne, Belgium; Philippe Minette, Centres Hospitaliers Jolimont, Belgium; Jean-Marc Minon, Centre Hospitalier Régional de la Citadelle, Liège, Belgium; Saphia Mokrane, Hôpitaux Iris Sud, Brussels, Belgium; Catherine Nachtergal, Cliniques de l'Europe, Brussels, Belgium; Séverine Noirhomme, Centre Hospitalier Régional de Namur, Belgium; Denis Piérard, Universitair Ziekenhuis Brussel, Brussels; Camelia Rossi, Centre Hospitalier Universitaire Ambroise Paré, Mons, Belgium; Carole Schirvel, CHIREC, Brussels, Belgium; Erica Sermijn, A.S.Z. Ziekenhuis, Aalst, Belgium; Ben Serrien, Sciensano, Belgium; Fabio Taccone, Hôpital Erasme, Brussels, Belgium; Frank Staelens, OLV Ziekenhuis, Aalst, Belgium; Filip Triest, Algemeen Ziekenhuis Sint Lucas, Gent, Belgium; Nina Van Goethem, Sciensano, Brussels, Belgium; Jens Van Praet, Algemeen Ziekenhuis Sint Jan, Brugge-0ostende, Belgium; Anke
Vanhoenacker, Ziekenhuisnetwerk Antwerpen, Belgium; Roeland Verstraete, Algemeen Ziekenhuis, Monica, Belgium; Elise Willems, Algemeen Ziekenhuis Nikolaas, Sint-Niklaas, Belgium; Chloé Wyndham-Thomas, Sciensano, Belgium.

Contributors JP, MABvdS, M-AW and DVB conceptualised the study. DVB performed data curation and provided data. JP, EG, EM and DS developed methodology and performed analysis. JP supervised the study and drafted the manuscript. All authors have critically reviewed, commented and approved the manuscript before submission.

Funding The authors have not declared a specific grant for this research from any funding agency in the public, commercial or not-for-profit sectors.

Competing interests None declared.

Patient consent for publication Not required.

Provenance and peer review Not commissioned; externally peer reviewed.

Data availability statement Data are available upon reasonable request. The data that support the findings of this study are available from Sciensano but restrictions apply to the availability of these data, which were used under license for the current study, and so are not publicly available. Data are however available from the authors upon reasonable request and with permission of Sciensano.

Supplemental material This content has been supplied by the author(s). It has not been vetted by BMJ Publishing Group Limited (BMJ) and may not have been peer-reviewed. Any opinions or recommendations discussed are solely those of the author(s) and are not endorsed by BMJ. BMJ disclaims all liability and responsibility arising from any reliance placed on the content. Where the content includes any translated material, BMJ does not warrant the accuracy and reliability of the translations (including but not limited to local regulations, clinical guidelines, terminology, drug names and drug dosages), and is not responsible for any error and/or omissions arising from translation and adaptation or otherwise.

Open access This is an open access article distributed in accordance with the Creative Commons Attribution Non Commercial (CC BY-NC 4.0) license, which permits others to distribute, remix, adapt, build upon this work non-commercially, and license their derivative works on different terms, provided the original work is properly cited, appropriate credit is given, any changes made indicated, and the use is non-commercial. See: http://creativecommons.org/licenses/by-nc/4.0/.

ORCID iDs

José L. Peñalvo http://orcid.org/0000-0003-0096-6238

Elly Mertens http://orcid.org/0000-0003-4957-4235

Dominique Van Beckhoven http://orcid.org/0000-0003-0821-2756

\section{REFERENCES}

1 Yang X, Yu Y, Xu J, et al. Clinical course and outcomes of critically ill patients with SARS-CoV-2 pneumonia in Wuhan, China: a singlecentered, retrospective, observational study. Lancet Respir Med 2020;8:475-81.

2 Guan W-J, Ni Z-Y, Hu Y, et al. Clinical characteristics of coronavirus disease 2019 in China. N Engl J Med 2020;382:1708-20.

3 Zhang J-J, Dong X, Cao Y-Y, et al. Clinical characteristics of 140 patients infected with SARS-CoV-2 in Wuhan, China. Allergy 2020;75:1730-41.

4 Kassir R. Risk of COVID-19 for patients with obesity. Obes Rev 2020;21:e13034.

5 Petrilli CM, Jones SA, Yang J, et al. Factors associated with hospital admission and critical illness among 5279 people with coronavirus disease 2019 in New York City: prospective cohort study. BMJ 2020;369:m1966.

6 Wan Y, Shang J, Graham R, et al. Receptor recognition by the novel coronavirus from Wuhan: an analysis based on decade-long structural studies of SARS coronavirus. J Virol 2020;94:e00127-20.

7 Rossi GP, Sanga V, Barton M. Potential harmful effects of discontinuing ACE-inhibitors and ARBs in COVID-19 patients. Elife 2020;9:e57278.

8 Ishiyama Y, Gallagher PE, Averill DB, et al. Upregulation of angiotensin-converting enzyme 2 after myocardial infarction by blockade of angiotensin II receptors. Hypertension 2004;43:970-6.

9 Patel AB, Verma A. COVID-19 and angiotensin-converting enzyme inhibitors and angiotensin receptor blockers: what is the evidence? JAMA 2020;323:1769-70.

10 Emilsson V, Gudmundsson EF, Aspelund T. Antihypertensive medication uses and serum ACE2 levels. medRxiv2020;20108738. 
11 Jiang X, Eales JM, Scannali D, et al. Hypertension and reninangiotensin system blockers are not associated with expression of angiotensin-converting enzyme 2 (ACE2) in the kidney. Eur Heart $J$ 2020;41:4580-8.

12 Sama IE, Ravera A, Santema BT, et al. Circulating plasma concentrations of angiotensin-converting enzyme 2 in men and women with heart failure and effects of renin-angiotensin-aldosterone inhibitors. Eur Heart J 2020;41:1810-7.

13 Zheng Y-Y, Ma Y-T, Zhang J-Y, et al. COVID-19 and the cardiovascular system. Nat Rev Cardiol 2020;17:259-60.

14 Fang L, Karakiulakis G, Roth M. Are patients with hypertension and diabetes mellitus at increased risk for COVID-19 infection? Lancet Respir Med 2020;8:e21.

15 Peng YD, Meng K, Guan HQ, et al. [Clinical characteristics and outcomes of 112 cardiovascular disease patients infected by 2019nCoV]. Zhonghua Xin Xue Guan Bing Za Zhi 2020;48:450-5.

16 Huang Z, Cao J, Yao Y, et al. The effect of RAS blockers on the clinical characteristics of COVID-19 patients with hypertension. Ann Transl Med 2020;8:430.

$17 \mathrm{Li} \mathrm{J}$, Wang X, Chen J, et al. Association of renin-angiotensin system inhibitors with severity or risk of death in patients with hypertension hospitalized for coronavirus disease 2019 (COVID-19) infection in Wuhan, China. JAMA Cardiol 2020;5:825-30.

18 Meng X, Liu Y, Wei C, et al. Angiotensin converting enzyme inhibitors and angiotensin receptor blockers improved the outcome of patients with severe COVID-19 and hypertension. Sci China Life Sci 2021;64:836-9.

19 Feng Y, Ling Y, Bai T, et al. COVID-19 with different severities: a multicenter study of clinical features. Am J Respir Crit Care Med 2020;201:1380-8.

20 Yang G, Tan Z, Zhou L, et al. Effects of angiotensin II receptor blockers and ACE (angiotensin-converting enzyme) inhibitors on virus infection, inflammatory status, and clinical outcomes in patients with COVID-19 and hypertension: a single-center retrospective study. Hypertension 2020;76:51-8.

21 Zhang J, Wang M, Ding W, et al. The interaction of RAAS inhibitors with COVID-19: current progress, perspective and future. Life Sci 2020;257:118142.

22 Mancia G, Rea F, Ludergnani M, et al. Renin-AngiotensinAldosterone system blockers and the risk of Covid-19. N Engl J Med 2020;382:2431-40.

23 de Abajo FJ, Rodríguez-Martín S, Lerma V, et al. Use of reninangiotensin-aldosterone system inhibitors and risk of COVID-19 requiring admission to hospital: a case-population study. Lancet 2020;395:1705-14.

24 Reynolds HR, Adhikari S, Pulgarin C, et al. Renin-AngiotensinAldosterone system inhibitors and risk of Covid-19. N Engl J Med 2020;382:2441-8.

25 Jung S-Y, Choi JC, You S-H, et al. Association of renin-angiotensinaldosterone system inhibitors with COVID-19-related outcomes in Korea: A nationwide population-based cohort study. Clin Infect Dis 2020;71:2121-8.

26 Trifiro G, Massari M, Da Cas R. Renin-angiotensin-aldosterone system inhibitors and risk of death in patients hospitalised with COVID-19: a retrospective Italian cohort study of 43,000 patients. Drug Saf2020:1-12 (published Online First: 2020/08/28).

27 Zhang P, Zhu L, Cai J, et al. Association of inpatient use of angiotensin-converting enzyme inhibitors and angiotensin II receptor blockers with mortality among patients with hypertension hospitalized with COVID-19. Circ Res 2020;126:1671-81.

28 Gao C, Cai Y, Zhang K, et al. Association of hypertension and antihypertensive treatment with COVID-19 mortality: a retrospective observational study. Eur Heart J 2020;41:2058-66.

29 Fosbøl EL, Butt JH, Østergaard L, et al. Association of angiotensinconverting enzyme inhibitor or angiotensin receptor blocker use with COVID-19 diagnosis and mortality. JAMA 2020;324:168-77.

30 Hippisley-Cox J, Young D, Coupland C, et al. Risk of severe COVID-19 disease with ACE inhibitors and angiotensin receptor blockers: cohort study including 8.3 million people. Heart 2020;106:1503-11.

31 Fernández-Ruiz I. RAAS inhibitors do not increase the risk of COVID-19. Nat Rev Cardiol 2020;17:383.
32 Jarcho JA, Ingelfinger JR, Hamel MB, et al. Inhibitors of the reninangiotensin-aldosterone system and Covid-19. N Engl J Med 2020;382:2462-4.

33 Wolkewitz M, Lambert J, von Cube M, et al. Statistical analysis of clinical COVID-19 data: a Concise overview of lessons learned, common errors and how to avoid them. Clin Epidemiol 2020;12:925-8.

34 Cohen JB, D'Agostino McGowan L, Jensen ET, et al. Evaluating sources of bias in observational studies of angiotensin-converting enzyme inhibitor/angiotensin II receptor blocker use during COVID-19: beyond confounding. J Hypertens 2021;39:795-805.

35 Hazard D, Kaier K, von Cube M, et al. Joint analysis of duration of ventilation, length of intensive care, and mortality of COVID-19 patients: a multistate approach. BMC Med Res Methodol 2020;20:206.

36 von Cube M, Grodd M, Wolkewitz M, et al. Harmonizing heterogeneous endpoints in coronavirus disease 2019 trials without loss of information. Crit Care Med 2021;49:e11-19.

37 Van Goethem N, Vilain A, Wyndham-Thomas C, et al. Rapid establishment of a national surveillance of COVID-19 hospitalizations in Belgium. Arch Public Health 2020;78:121.

38 WHO. COVID-19 and NCDs, 2020. Available: https://www.who.int/ internal-publications-detail/covid-19-and-ncds [Accessed $27 \mathrm{Mar}$ 2020].

39 de Azambuja E, Brandão M, Wildiers $\mathrm{H}$, et al. Impact of solid cancer on in-hospital mortality overall and among different subgroups of patients with COVID-19: a nationwide, population-based analysis. ESMO Open 2020;5:e000947.

40 Catteau L, Dauby N, Montourcy M, et al. Low-dose hydroxychloroquine therapy and mortality in hospitalised patients with COVID-19: a nationwide observational study of 8075 participants. Int J Antimicrob Agents 2020;56:106144.

41 Survival R package. Available: https://cran.r-project.org/web/ packages/survival/survival.pdf [Accessed 1 Nov 2020].

42 de Wreede LC, Fiocco M, Putter H. The mstate package for estimation and prediction in non- and semi-parametric multi-state and competing risks models. Comput Methods Programs Biomed 2010;99:261-74.

43 Mstate R package. Available: https://cran.r-project.org/web/ packages/mstate/mstate.pdf [Accessed 1 Nov 2020].

44 Taccone FS, Vangoethem N, Depauw R. The role of organizational characteristics on the outcome of COVID-19 patients admitted to the ICU in Belgium. Lancet Regional Health - Europe 2020:100019.

45 Danser AHJ, Epstein M, Batlle D. Renin-Angiotensin system blockers and the COVID-19 pandemic: at present there is no evidence to abandon renin-angiotensin system blockers. Hypertension 2020;75:1382-5.

46 Lahens A, Mullaert J, Gressens S, et al. Association between renin-angiotensin-aldosterone system blockers and outcome in coronavirus disease 2019: analysing in-hospital exposure generates a biased seemingly protective effect of treatment. $J$ Hypertens 2021;39:367-75.

47 Cannata F, Chiarito M, Reimers B, et al. Continuation versus discontinuation of ACE inhibitors or angiotensin II receptor blockers in COVID-19: effects on blood pressure control and mortality. Eur Heart J Cardiovasc Pharmacother 2020;6:412-4.

48 de Abajo FJ, Rodríguez-Miguel A, Rodríguez-Martín S, et al. Impact of in-hospital discontinuation with angiotensin receptor blockers or converting enzyme inhibitors on mortality of COVID-19 patients: a retrospective cohort study. BMC Med 2021;19:118.

49 Cohen JB, Hanff TC, William P, et al. Continuation versus discontinuation of renin-angiotensin system inhibitors in patients admitted to hospital with COVID-19: a prospective, randomised, open-label trial. Lancet Respir Med 2021;9:275-84.

50 Lopes RD, Macedo AVS, de Barros E Silva PGM, et al. Effect of discontinuing vs continuing angiotensin-converting enzyme inhibitors and angiotensin II receptor blockers on days alive and out of the hospital in patients admitted with COVID-19: a randomized clinical trial. JAMA 2021;325:254-64.

51 Meira-Machado L, de Uña-Alvarez J, Cadarso-Suárez C, et al. Multistate models for the analysis of time-to-event data. Stat Methods Med Res 2009;18:195-222.

52 Putter H, Fiocco M, Geskus RB. Tutorial in biostatistics: competing risks and multi-state models. Stat Med 2007;26:2389-430. 